\title{
Nanomaterials in Operando Conditions
}

\section{Dario Stacchiola}

\section{Brookhaven National Laboratory, Upton, New York, United States}

Heterogeneous catalysts undergo changes in their structure as they mediate a chemical reaction. Multiple experimental approaches have been developed to understand these changes. Due to their structural and chemical complexity, and the difficulty of interrogating them under reaction conditions, catalysts are generally characterized before and after chemical reactions. Thus, dynamic active phases formed in catalysts under reaction conditions, which generate nanometer sized multifunctional active centers at metal/oxide/modifiers interfaces, and the associated reaction mechanisms are generally unknown. Practical catalysts with a porous framework, such as zeolites, host catalytic reactions at active sites engrained in the pores and channels of the scaffold. The mechanism of interaction at these active sites, defining catalyst performance, remains elusive, in large part due to the lack of surface characterization methods available for thick films or powders. Since the structure of catalysts change as reacting molecules interact with it in route to forming products, only in-situ techniques allow following the dynamic processes involved on the surface of a catalyst. There has been considerable progress in the development and use of surface science techniques to follow catalytic reactions in-situ. Photon-in/photon-out techniques, such as IR spectroscopy, were used early on but photo-electron spectroscopic techniques, where electrons interact strongly with the reactants in the gas phase, took much longer to become widely available. Electron based structural techniques, such as scanning tunneling microscopy (STM), are still only available on a limited number of laboratories. In this presentation, I will highlight case studies showing how complementary insitu techniques including ambient pressure (AP) X-ray photoelectron spectroscopy (AP-XPS), infrared reflection absorption spectroscopy (AP-IRRAS) and AP-STM can be applied to study heterogeneous interfaces in atomically-well-defined model catalysts.

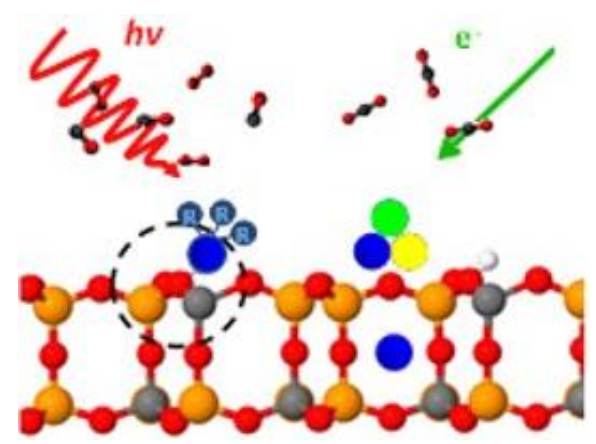

Figure 1. In-situ and Operando Multimodal characterization of catalysts

\section{References}

"Reversible oxidation and reduction of gold-supported iron oxide islands at room temperature"

J. Chem. Phys. 152, 074710 (2020)

"Single Atoms in Nano-Cages: Immobilization of Ar Atoms in Two-Dimensional Zeolite Models" Nature Communications 8, 16118 (2017)

"Highly Active Copper-Ceria and Copper-Ceria-Titania Catalysts for Methanol Synthesis from $\mathrm{CO}_{2}$ " Science 345, 546-550 (2014) 